\title{
ESTIMATION OF GLUCOSE LEVELS IN BLOOD AND SALIVA-A COMPARATIVE STUDY
}

\author{
Gayathri Karthikeyan*1, GifrinaJayaraj ${ }^{2}$ and K.R.Don $^{3}$ \\ ${ }^{1}$ I BDS, Saveetha Dental College \& Hospitals, Poonamalle High Road, Chennai, \\ ${ }^{2}$ Department of Oral and maxofacial pathology Saveetha Dental College \& Hospitals, \\ Poonamalle High Road, Chennai, \\ ${ }^{3}$ Department of Oral andmaxofacial pathology, Saveetha Dental College \& Hospitals, \\ Poonamalle High Road, Chennai,
}

\section{A R T I C L E I N F O}

\section{Article History:}

Received $18^{\text {th }}$ December, 2016

Received in revised form $16^{\text {th }}$ January, 2017

Accepted $26^{\text {th }}$ February, 2017

Published online $28^{\text {th }}$ March, 2017

\section{Key words:}

Glucose Levels, Blood Collection

\begin{abstract}
A B S T R A C T
BACKGROUND: Blood is the most common biological fluid used in investigatory projects but it is an invasive procedure, saliva is another biological fluid that can be used as an alternative to blood since it is less invasive and most of the biological parameters found using blood can also be identified in saliva

AIM: to estimate the glucose levels in blood and saliva and verify if there is a correlation between them.

OBJECTIVE: to compare the glucose levels in blood and saliva and estimate the level and justify that the levels in both are equal

METHOD: The study group comprised of 25 randomly selected patients. Blood collection: The samples were collected using the venipuncture methods. Values were calculated using a semi auto analyzer to estimate the random blood glucose levels. Saliva collection: Mostly only stimulated saliva was collected from the subjects. The saliva was collected by asking the participants to spit into the eppendorf, after rinsing their mouth. Statistical analysis: Pearson's correlation test was done on the data collected and it showed a negative correlation.

RESULT: When the statistical analysis was done it showed a negative correlation in the study, which means that if salivary glucose levels decreases the blood glucose levels increase.

CONCLUSION: The present study sheds some light on the importance of early diagnosis and preferment and utilization of non-invasive saliva analysis over the invasive blood analysis. Even though the present study shows a negative correlation more research should be conducted in this area.
\end{abstract}

Copyright $\odot 2017$ Gayathri Karthikeyan et al. This is an open access article distributed under the Creative Commons Attribution License, which permits unrestricted use, distribution, and reproduction in any medium, provided the original work is properly cited.

\section{INTRODUCTION}

Diabetes mellitus is a clinically and genetically heterogeneousmetabolic disease characterized by hyperglycemia and deregulationof carbohydrate, protein, and lipid metabolism. The primary feature of diabetes mellitus is chronic hyperglycemia, resulting from either a defect in insulin secretion from pancreas or resistance of body's cells to insulin action or both (1).

The global prevalence of diabetes is $6.4 \%$ in adult population. Worldwide, the number of people with diabetes is expected to grow to 438 million by 2030 , corresponding to $7.8 \%$ of the adult population.

*Corresponding author: Gayathri Karthikeyan I BDS, Saveetha Dental College \& Hospitals, Poonamalle High Road, Chennai,
The crude prevalence rate of diabetes in urban areas is about $9 \%$ and in rural areas, has increased to around $3 \%$ of the total population (2).Type 2 diabetes mellitus is the fifth most common condition and the sixth leading cause of mortality amongst the elderly (3).

FalaceMarcheti in 1989 and Amer in 2001 reported similar results. But, Leach in 1970 and Ficara in 1975 did not find any significant relationship between blood glucose and salivary glucose level in diabetic patients (5).Owing to lack of sufficient diagnosis and treatment, diabetes is a major cause of death worldwide, more than half of the diabetics remain undiagnosed especially the patients with Type 2 diabetes. Monitoring people with diabetes by repeatedly estimating blood glucose and glycosylated hemoglobin levels is invasive which becomes appalling and expensive over time (3).

The need to monitor periodic blood glucose is present because it reveals the pattern of blood glucose change in individuals, 
and helps in planning of meals, and also at what time of the day a patient has to take medication. The choice of blood as a diagnostic fluid for clinical testing is clear-cut considering its close relationship to the homeostasis of the body (4) .While the early diagnosis of diabetes is essential toprevent its devastating complications, the currentmethod of investigation needs the painful needleprickto withdraw blood, which may discouragethe individuals from the investigation (2).

Saliva fulfills several of the chief diagnostic concerns for a diagnostic bio fluid as it is obtained noninvasively, and its collection requires no special skill (4) Saliva acts as a mirror of the body and, hence, is a perfect medium to be explored for disease and health surveillance (6). This study was conducted to check if saliva can be used as an alternative bio-fluid for glucose estimation.

\section{MATERIALS AND METHODS}

$>$ Sample size: The study group comprised of 25 randomly selected patients. Almost all the subjects were non diagnosed patients. The study comprised of 9 male participants and 16 female participants

$>$ Age group: aged between 18 to 80 years.

Sample collection:

$>$ Blood collection: The samples were collected using the venipuncture methods. Values were calculated using a semi auto analyzer to estimate the random blood glucose levels. The method that was used to estimate the serum glucose level was Hexokinasemethod.

> Saliva collection: Mostly only stimulated saliva was collected from the subjects. The saliva was collected by asking the participants to spit into the eppendorf, after rinsing their mouth well. The random saliva glucose values were calculated using the glucose oxidase method and the values were calculated using a calorimeter.

$>$ Statistical analysis: Pearson's correlation test was done on the data collected and it showed a negative correlation

\section{RESULT}

$>$ The correlation coefficient of the following data is 0.1163187

$>3$ people had elevated levels of blood glucose but their salivary glucose levels were relatively very low.

$>\quad$ The mean of blood glucose levels was 108.92 and that of saliva was 9.128 , this showed a very high variance.

$>$ When the statistical analysis was done it showed a negative correlation in the study, which means that if salivary glucose levels decreases the blood glucose levels increase.

$>$ The salivary glucose levels are inversely proportional to each other and since it is giving a negative value the results were insignificant for random BGL and SGL.

\begin{tabular}{|c|c|c|}
\hline PATIENT & $\begin{array}{c}\text { BLOOG(G) } \\
(\mathrm{mg} / \mathrm{dl})\end{array}$ & $\begin{array}{c}\text { SALIVA(G) } \\
(\mathrm{mg} / \mathrm{dl})\end{array}$ \\
\hline $\mathrm{P} 1$ & 94 & 51.51 \\
\hline $\mathrm{P} 2$ & 87 & 27.27 \\
\hline P3 & 85 & 3.03 \\
\hline $\mathrm{P} 4$ & 117 & 45.45 \\
\hline P5 & 96 & 3.03 \\
\hline P6 & 97 & 3.03 \\
\hline P7 & 116 & 3.03 \\
\hline P8 & 98 & 27.27 \\
\hline P9 & 90 & 0 \\
\hline $\mathrm{P} 10$ & 97 & 6.06 \\
\hline P11 & 96 & 3.03 \\
\hline P12 & 93 & 12.12 \\
\hline P13 & 256 & 0 \\
\hline P14 & 82 & 0 \\
\hline P15 & 83 & 3.03 \\
\hline P16 & 105 & 9.09 \\
\hline P17 & 134 & 0 \\
\hline P18 & 190 & 12.12 \\
\hline P19 & 113 & 9.09 \\
\hline P20 & 87 & 3.03 \\
\hline P21 & 67 & 2 \\
\hline P22 & 106 & 0 \\
\hline $\mathrm{P} 23$ & 145 & 4 \\
\hline $\mathrm{P} 24$ & 80 & 1 \\
\hline $\mathrm{P} 25$ & 109 & 0 \\
\hline
\end{tabular}

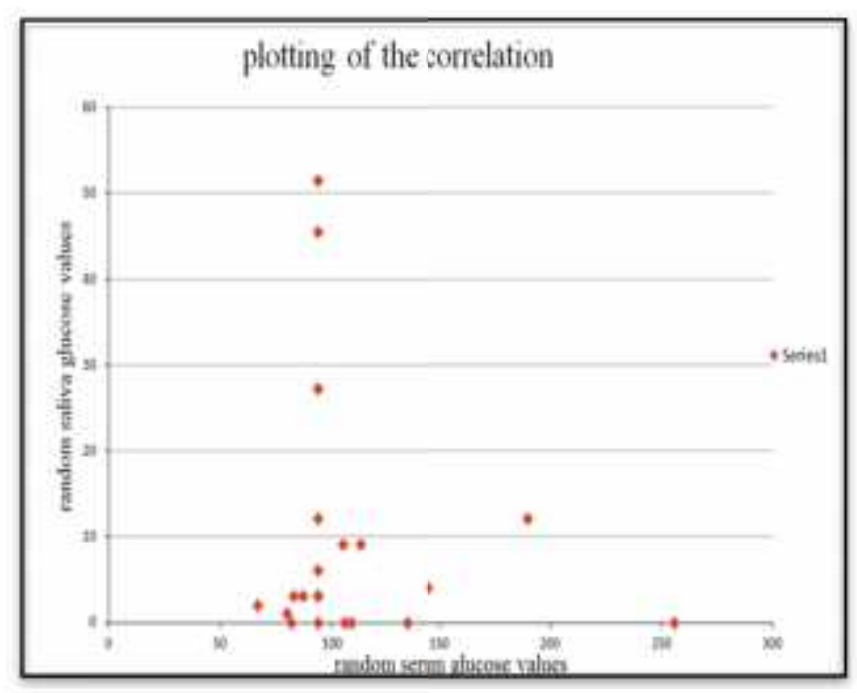

\section{DISCUSSION}

$>$ From the data collected it was observed that 3 individuals had elevated blood glucose levels but their their salivary glucose level was relatively low, this was because of the negative correlation and so the salivary and blood glucose levels were inversely proportion to each other. Patient number 13 had a blood glucose value of $256 \mathrm{mg} / \mathrm{dl}$ where as he had a salivary glucose value of $0 \mathrm{mg} / \mathrm{dl}$ showing the 
inverse proportionality. In this study, one of the main reason that there was a negative correlation is because the sample Size was less also another reason that the results were negative was that there are microbes in the mouth that feed on the salivary glucose making the value calculation harder. If the sample size was increased their could be a positive correlation.

$>$ Data indicates that in 2011, 366 million people worldwide were affected by diabetes and the number is continuing to climb steeply.By 2030, predictions suggest that the number of people with diabetes will reach 552 million (7).The correlation between SGL and BGL is supported by various studies .whilst other study outcomes were in contradiction to this few researches have studied correlation between SGL and BGL, suggesting the paucity of literature on this aspect (2). Diabetes mellitus is certain to be one of the fundamental challenges that is faced by any health care professional in the present day. The two key aspects of diabetic management are normalization of blood glucose level and its judicious monitoring; both of these need the patient's regular compliance. It is utmost important for diabetics to maintain the blood glucose level to normal throughout their lifespan as that indeed has impact on the risk of development of complications (2).

$>$ The present method of blood glucose estimation needs the venepuncture, which is highly traumatic to the patients at times, especially to the children. Apart from physical trauma, process also renders mental trauma and anxiety about the procedure to discourage the patients further. (2) Regarding the SG

$>$ Concentrations, the results obtained in different studies are varied due to diversity of selection criteria / sampling / study population. (3) The study found no correlation between salivary glucose and sex. It is high time that is problem comes to light and some solution has to been found soon so as to make the estimation of glucose levels more easy so that patients will not hamper their regular visit to diabetic clinic.

$>$ Saliva is said to be the ultra-filtrate of blood. Glucose is one of the blood components that are transferable across the salivary gland epithelium in proportion to its concentration in blood. Secondly, whole saliva is the biologic fluid that is simple to collect. The correlation between SGL and BGL cannot be justified until the salivary glucose estimation is authenticated. There should be homogeny in the methodology used plus the study samplings need to be standardized to have the study outcomes which will be comparable and sustainable. (2)
$>$ There have been varying reports on the salivary glucose levels based on gender. Darwazeh et al found higher levels of salivary glucose in males as compared to females, and Soares et al did not find any significant relationship with gender (4).FalaceMarcheti in 1989 and Amer in 2001 reported similar results. But, Leach in 1970 and Ficara in 1975 did not find any significant relationship between blood glucose and salivary glucose level in diabetic patients.(5)Statistically nonsignificant correlations between blood and salivary glucose in the study and control groups were obtained by Tenovuo et al.,Aryeh et al., and Vaziri et al., This variation in result might have been because of the small sample size.(6) Englander et al expressed doubt regarding replacement of plasma with parotid secretion in the diagnosis of diabetes mellitus, because of its lower levels of glucose concentration(7) A study carried out in last few years revealed that the salivary samples of the non-diabetic control subjects did not show the presence of glucose even in the slightest concentrations.(8)

$>$ There is still hope and lot of research has been going on to find an alternative to blood for diagnostic investigations. If saliva is soon proven to be an excellent bio-fluid for diagnostics tests then it would be less expensive noninvasive and simple to collect as well this would be easierfor juveniles with diabetes.Moreover, it will be possible for the people with diabetes to self-monitor themselves with method that is easier and not painful (2). The present study sheds some light on the importance of early diagnosis and preferment and utilization of noninvasive saliva analysis over the invasive blood analysis.

\section{REFERENCES}

1. M Dhanya, S Hegde,Salivary glucose as a diagnostic tool in Type II diabetes mellitus: A case-control study, Department of Oral Medicine, The Oxford Dental College and Hospital, Bengaluru, Karnataka, India, 02-Nov-2015

2. AnupamaHegde, RamyaShenoy, Prajwal D'Mello, Smitha A , Tintu A and PoornimaManjrekar , Alternative markers of glycemic status in diabetes mellitus, March 152010

3. Arati S. Panchbhai, Correlation of Salivary Glucose Level with Blood Glucose Level in Diabetes Mellitus,1Department of Oral Medicine and Radiology, SharadPawar Dental College and Hospital, DMIMS University, Sawangi- Meghe, Wardha, India, 4 July 2012.

4. Shruti Gupta, MDS, SimarpreetVirkSandhu, MDS, MAMS, HimantaBansal, MDS, DNB, and Deepti Sharma, MDS, Comparison of Salivary and Serum Glucose Levels in Diabetic Patients, 2014. 
5. A. Azizi , A. Modaberi, The Correlation of Blood Glucose with Salivary glucose Level in Diabetic Patients , 24 Jul 2012 , Accepted: 30 Sept 2013

6. Karandeep Singh Arora, NageshBinjoo, G. V. Ramachandra Reddy, Prabhpreet Kaur, RichaModgil, and Lalit Singh Negi ,Determination of normal range for fasting salivary glucose in Type 1 diabetics, 2015.

7. Panda Abikshyeet, Venkatapathy Ramesh, NirimaOza, Glucose estimation in the salivary secretion of diabetes mellitus patients, 2012.

8. Agrawal RP1, Sharma N2, Rathore MS, Gupta VB, Jain S, Agarwal V and Goyal Noninvasive Method for Glucose Level Estimation by Saliva, 2013.

9. Englander HR, Jeffay AI, Fuller JB, Chauncey HH (1963) glucose oncentrations in blood plasma and parotid saliva of individuals with and without diabetes mellitus. J Dent Res 42: 1246.

10. Amer S, Yousuf M, Siddqiui PQ, Alam J (2001) Salivary glucose oncentrations in patients with diabetes mellitus--a minimally invasive technique for monitoring blood glucose levels.

11. Naik VV, Satpathy Y, Pilli GS, Mishra MN. Comparison and correlation of glucose levels in serum and saliva of patients with diabetes mellitus. Indian J Pub Health Res Dev. 2011;
12. Sashikumar R, Kannan R. Salivary glucose levels and oral candida carriage in type II diabetics. Oral Surg Oral Med Oral Pathol Oral RadiolEndod. 2010.

13. Vasconcelos AC, Soares MS, Almeida PC, Soares TC. Comparative study of the concentration of salivary and blood glucose in type 2 diabetic patients. J Oral Sci. 2010 June;

14. Reuterving CO, Reuterving E, Hagg E, Ericson T. Salivary flor rate and salivary glucose concentration in patients with diabetes mellitus influence of severity of diabetes. DiabMetabol.1987 July-August;

15. Sreedevi, Shashikanth MC, Shambulingappa P. Comparison of serum glucose and salivary glucose in diabetic patients. Journal of Indian Academy of Oral Medicine and Radiology. 2008

16. Panchbhai AS. Correlation of salivary glucose level with blood glucose level in diabetes mellitus. J Oral Maxillofac Res. 2012.

17. Forbat LN, Collins RE, Maskell GK, Sönksen PH. Glucose concentrations in parotid fluid and venous blood of patients attending a diabetic clinic. J R Soc Med. 1981.

\section{Please cite this article in press as:}

Gayathri Karthikeyan, GifrinaJayaraj and K.R.Don (2017), Estimation of glucose levels in blood and saliva-a comparative study, International Journal of Current Advanced Research, 6(03), pp. 2992-2995.

http://dx.doi.org/10.24327/ijcar.2017.2995.0162 